Natural Hazards and Earth System Sciences, 5, 495-498, 2005

SRef-ID: 1684-9981/nhess/2005-5-495

European Geosciences Union

(c) 2005 Author(s). This work is licensed

under a Creative Commons License.

\title{
Quantitative expression on drought magnitude and disaster intensity
}

\author{
L. H. Feng ${ }^{1}$ and X. C. Zhang ${ }^{2}$ \\ ${ }^{1}$ Department of Geography, Zhejiang Normal University, Jinhua 321004, China \\ ${ }^{2}$ Institute of Remote Sensing, Zhejiang Normal University, Jinhua 321004, China
}

Received: 16 December 2004 - Revised: 19 April 2004 - Accepted: 9 June 2005 - Published: 19 July 2005

\begin{abstract}
According to the calculation of wind scale and earthquake magnitude, this paper puts forward the concept of drought magnitude and disaster intensity and lays out a new method by quantitative calculation. Drought magnitude is the quantitative index that describes the scale of a drought, and disaster intensity of drought is the quantitative index to describe the loss caused by a drought. Both indices have many theoretical and practical advantages with definable concepts and simple applications; therefore they are of important practical significance.
\end{abstract}

\section{Introduction}

Different researchers have different thoughts on drought and drought disaster from different angles, and they give different definitions on drought and drought disaster so that there are more than 10 indices of drought used regularly both at home and abroad. These include meteorological drought index, hydrological drought index, agricultural drought index and economic drought index (Zhang, 1997).

In fact, drought and drought disaster are two different concepts in science. By analyzing the variety of drought definitions, we come to the conclusion that drought is a climatic phenomenon where less than normal precipitation occurs within a specified period of time (Sauchyn et al., 2003; Sevinc and Zekai, 2003). It is a natural phenomenon, and drought itself doesn't equal disaster. Only when drought causes damage to human social and economic activity does it become drought disaster, just as an earthquake in a place without human beings is just a natural phenomenon without disaster.

To understand and communicate the information of drought in a specific area on a timely basis, two quantitative indices are needed, drought magnitude and disaster intensity, as well as a quantitative calculation method. Of course,

Correspondence to: L. H. Feng

(fenglh@zjnu.cn) the calculated drought magnitude and disaster intensity must be capable of reflecting the true drought situation and easily communicating the information. Therefore, two principles for calculation of drought magnitude and disaster intensity must be followed: (1) the selected characteristic quantity must reflect the nature of the situation; (2) the magnitude calculated must be easy to remember. This paper attempts to conduct a specific analysis of this issue.

\section{Enlighten from wind speed and earthquake indices}

Comparatively, the quantitative indices wind scale and earthquake magnitude are used to reflect the scale of typhoons and earthquakes, and another quantitative index, earthquake intensity, is used to measure the disaster severity of earthquakes. Therefore the wind force of typhoon No. 0216 (Sinlaku) which landed in Cangnan, Zhejiang Province, China, 7 September 2002 can be described as force 12. The earthquake in Tangshan, China on 28 July 1976 can be described as a magnitude 7.8, and the disaster severity (earthquake intensity) can be defined as degree 11. However, the drought along the middle and down streams of the Yangtze River and the Yellow-Huai River Plain in 1978 can only be qualitatively described as "catastrophic drought" with "heavy losses" for the magnitude and the disaster severity respectively. Therefore, to parallel wind scale and earthquake magnitude, this study proposes two quantitative indices, drought magnitude and disaster intensity, to measure the magnitude and disaster situation of drought and a new method by quantitative calculation.

In the quantitative calculation of magnitude and disaster intensity, the selection of characteristic value is of utmost importance (Adnan, 2003; Bonaccorso and Bordi, 2003). In order to calculate the magnitude of wind force, British scholar F. Beaufort (1805) chose wind speed, which could best reflect the magnitude of wind force, and divided wind force into 13 magnitudes (magnitude $0 \sim 12$ ) (Wu and Zhang, 1986). The relationship between the magnitudes of wind 
force $F$ and wind speed $V(\mathrm{~m} / \mathrm{s})$ can be expressed approximately as:

$F=1.12 V^{2 / 3}$.

To calculate magnitude of earthquakes, American scholar C. F. Richter and B. Gutenberg (1935) chose the substantial characteristic value, the energy released by an earthquake, which could best reflect its magnitude, to establish the following relationship between earthquake magnitude $M$ and energy $E(\mathrm{j})$ (Xu and Zhou, 1982):

$\lg E=11.8+1.5 M$.

It is widely recognized that the simpler the number designated to a magnitude, the easier for it to be remembered and disseminated. For instance, the wind speed of a typhoon is $V=32.7 \sim 36.9 \mathrm{~m} / \mathrm{s}$, and the energy of an earthquake is $E=6.3 \times 10^{16} \mathrm{j}$. These numeric values are too big and complicated for ordinary people, and even professionals have difficulty in understanding and remembering them. However, when wind speed $V=32.7 \sim 36.9 \mathrm{~m} / \mathrm{s}$ is converted to wind force $F=12$, and $E=6.3 \times 10^{16} \mathrm{j}$ is converted to earthquake magnitude $M=8$, they are widely accepted. One of the important reasons is that complicated numbers are converted into simple and meaningful value. In fact, wind speed and energy, wind scale and earthquake magnitude are all numerical codes, the important difference between them is that the later is a simple and easy concept.

The calculation of wind speed and earthquake magnitude gives us a model for a simple quantitative calculation method to express drought magnitude and disaster intensity.

\section{Quantitative expression of the magnitude of drought}

As mentioned previously, our definition of drought is a climatic phenomenon where less than normal precipitation occurs. Taking into account this definition and principle Eq. (1), we hold that the substantial characteristic values which best reflect the magnitude of drought are two elements of drought, namely lack of precipitation and duration of drought, as they are the indicators of drought scale and extent of destruction. The common notion that drought along the Yangtze and Yellow-Huai Rivers in 1978 was more severe than that of Henan Province in 1942, it mainly refers to the precipitation levels of the former being less than the latter, and the time of the former being longer compared with that of the latter. Therefore, this paper proposes precipitation and duration as the characteristic values for the calculation of drought magnitude.

Principle (2) implies that the simpler the number designated to a drought magnitude, the easier for it to be remembered and disseminated. Taking into consideration Principle (2) and the 13 wind scales and 9 earthquake scales, this study adopts ten numbers from 1 to 10 to describe the magnitude of drought.

From the formula structure of wind scale and earthquake magnitude, the relationship between characteristic value and magnitude is expressed by power function or logarithmic function. In order to have a similar structure in formula between drought magnitude and wind scale and earthquake magnitude, this paper adopts the following logarithmic function for calculation:

$N=a \lg K T+b$.

Where $N$ is drought magnitude (magnitude); $K$ is anomalous percentage of precipitation (\%); $T$ is the length of time of drought (d); and $a, b$ are undetermined parameters. In Eq. (3), $K$ is positive: $K=\left|\left(R_{i}-\bar{R}\right) / \bar{R} \times 100 \%\right|, R_{i}$ is the precipitation during the drought, $\bar{R}$ is the average precipitation over many years. Generally, $T$ uses the time period when precipitation falls below a certain standard, or the time interval between two rains. It is determined in accordance with the actual measured data from weather stations or historical records (Local Records etc.).

To distribute the calculated drought magnitudes within the range of 1-10, through intensive analysis of the distribution of anomaly percentage of precipitation $K$ as well as the duration $T$ of both domestic and foreign droughts, we propose that when $K=70 \%$ and $T=90$ d, $N=$ magnitude 6 ; when $K=20 \%$ and $T=30$ d, $N=$ magnitude 2 . When put into Eq. (3):

$\left\{\begin{array}{l}6=a \lg 70 \times 90+b \\ 2=a \lg 20 \times 30+b\end{array}\right.$

the results are $a \approx 4$ and $b \approx-9$. Therefore

$N=4 \lg K T-9$.

Meanwhile, we propose that when $N \geq 8$, it's a catastrophic drought; when $6 \leq N<8$, it's a severe drought; when $4 \leq N<6$, a medium drought; and when $N<4$, a mild drought.

Table 1 shows the two measurements of droughts as well as their magnitudes calculated from Eq. (4). From the table we can see that there was a severe drought of magnitude 6.8 in Luoyang City, Henan Province during January to September 1942, and a catastrophic drought of magnitude 8.2 in Nanjing City in 1978. In the second, third, and fourth years of Guangxu of the Qing Dynasty (1876 1878), there was drought in three successive years in provinces such as Henan, Hebei and Shanxi, with the worst being in 1877 affecting 308 counties. "Severe drought was all around Henan that year and there was no harvest in summer or autumn; bare land covered thousand li, and the hunger made people eat each other". According to the assumption of $K=60 \%$, the drought magnitude would be $N=10.3$ (catastrophic drought). Statistic shows that drought magnitude in the world is normally less than magnitude 10 .

\section{Quantitative expression of disaster intensity of drought}

Drought magnitude and disaster intensity are two separate concepts. The former is a natural phenomenon, while the later refers to the losses caused by the drought. Therefore we 
Table 1. Two elements of some droughts and their magnitudes.

\begin{tabular}{llccc}
\hline Year & Place & $\begin{array}{c}K \\
(\%)\end{array}$ & $\begin{array}{c}T \\
(\mathrm{~d})\end{array}$ & $\begin{array}{c}N \\
\text { (magnitude) }\end{array}$ \\
\hline $1876-1878$ & Henan Province & 60 & 1092 & 10.3 \\
1978 & Nanjing City & 55 & 364 & 8.2 \\
1922 & Bengbu City & 79 & 184 & 7.6 \\
1942 & Luoyang City & 32 & 275 & 6.8 \\
1899 & Chongqing City & 67 & 61 & 5.4 \\
1970 & Jinhua City & 95 & 31 & 4.9 \\
1894 & Beijing City & 54 & 31 & 3.9 \\
1969 & Ningbo City & 27 & 31 & 2.7 \\
\hline
\end{tabular}

define a drought disaster as: it is an event during which the precipitation has remained extremely scarce for a relatively long period (a few months to several years) so as to cause difficulty in meeting people's demands in life and production. Defining the disaster intensity of drought is similar to establishing the drought magnitude, and the afore-mentioned two principles shall be followed.

The disaster caused by a drought can be finally summarized into property loss. Where property loss includes both direct and indirect economic loss. Since it is difficult to estimate indirect economic loss, only direct economic loss is considered to calculate the disaster intensity of drought. Information on direct economic loss is easily acquired and can be used as the characteristic values to reflect the severity of drought disaster. The formula structure is:

$G=a \lg D+b$.

Where $G$ is the index expressing the severity of drought disaster, namely disaster intensity (degree); $D$ is direct economic loss ( $10^{4}$ yuan); $a$ and $b$ are undefined parameters.

To distribute the calculated drought disaster intensity within the range of $1-10$, through intensive analysis of drought disasters, we propose that when $D=10$ billion, $G=$ degree 8; when $D=0.1$ billion, $G=$ degree 4 . Putting these into Eq. (5), we get

$\left\{\begin{array}{l}8=a \lg 1000000+b \\ 4=a \lg 10000+b\end{array}\right.$,

the result of which is $a=2$ and $b=-4$. Then

$G=2 \lg D-4$.

In the meantime, we propose that when $G \geq 8$, it is a catastrophic disaster; when $6 \leq G<8$, it is a severe disaster; $4 \leq G<6$ equals a medium disaster; and $G<4$ signifies a minor disaster.

In the drought of Jinhua City, Zhejiang Province, China in 1990, there was a direct economic loss of 143 million yuan. According to the calculation from Eq. (6), the disaster intensity of the drought is $G=4.3$, or a medium disaster. The
1978 drought in China resulted in a direct economic loss of 14.6 billion yuan, raising the disaster intensity to degree 8.3, a catastrophic disaster. Statistic shows that the disaster intensity of drought in the world is normally less than degree 10 .

\section{Discussion}

From the relationship between drought magnitude and disaster intensity, generally speaking, the higher the drought magnitude is, the larger the disaster intensity is. For example, in 1978 , a catastrophic drought, with a magnitude 8.2, happened in Nanjing and the drought disaster intensity of that year in China reached degree 8.3, causing also immensely vast disaster. However, they are not always in proportion because their relationship is affected by population density, economic development, and the population's capability to mitigate damage. Therefore, droughts of the same magnitude result in different disaster intensities in different areas.

Drought index is commonly used for countries to disclose their drought situations. A $Z$ index is adopted in China Climatic Drought and Flood Journal (Ju et al., 1998):

$Z_{i}=\frac{6}{C_{s}}\left(\frac{C_{s}}{2} \varphi_{i}+1\right)^{1 / 3}-\frac{6}{C_{s}}+\frac{C_{s}}{6}$.

Where $C_{s}$ is a skewness coefficient, $\varphi_{i}$ is a standard variable. From this we can divide the drought in various areas in a year into four classes: (1) normal $(-0.84 \leq Z<0.84)$; (2) slight drought $(-1.44 \leq Z<-0.84)$; (3) medium drought $(-1.96 \leq Z<-1.44)$; (4) severe drought $(Z<-1.96)$.

From Eq. (7) we see that the structure of this $Z$-index formula is relatively complex, with large amounts of calculation. In comparison, the Eqs. (4) and (6) raised in this paper have the following advantages:

(1) The equations of drought magnitude and disaster intensity are widely applicable, and quantitative calculation and comparison can be made both in the same place and in different areas. 
(2) The equations of drought magnitude and disaster intensity are simple and are easy to calculate.

(3) The numeric values of drought magnitude and disaster intensity are simple, easily understood and remembered by ordinary people.

(4) The three values are substantial characteristic values that best reflect the drought magnitude and the disaster severity, and they are easy to acquire after a drought.

(5) Drought magnitude unifies the technical terms of magnitude in measuring natural variations such as drought, typhoon, earthquakes, and wave.

(6) Drought magnitude can provide certain theoretical bases for drought division.

(7) The disaster intensity of drought can guide the fight against disaster both at home and abroad.

(8) In drought forecasting, drought magnitude and disaster intensity can be used to alert and forecast the drought magnitude and the disaster situation in advance.

(9) Drought magnitude and disaster intensity can help people associate and presume the magnitude and the disaster situation of an on-going drought according to their memory.

\section{Conclusion}

This paper has put forward the concepts of drought magnitude and disaster intensity, and laid out a new method by quantitative calculation. Drought magnitude is the quantitative index that describes the scale of a drought, and disaster intensity of drought is the quantitative index to describe the scale of loss caused by a drought. There are many theoretical and practical advantages to describe the magnitude and the disaster situation of a drought by using the two indices respectively.
Drought magnitude and disaster intensity are two simple and easy quantitative indices with definable concepts and simple applications on judging drought magnitude and disaster situation. The recent increase of loss in drought makes it more important than ever to quantify drought magnitude and severity of disaster. These quantitative indices can be useful for proliferating the knowledge of drought in order to fight against disasters.

Edited by: M.-C. Llasat

Reviewed by: N. B. Gutman

\section{References}

Adnan, H. A.: Drought identification and characterization in Jordan, J. Arid. Env., 53, 585-606, 2003.

Bonaccorso, B. and Bordi, I.: Spatial variability of drought: an analysis of the SPI in Sicily, Water Re. Ma., 17, 273-296, 2003.

Ju, X. S., Zou, X. K., and Zhang Q.: The method of the climatic drought-flood index and its analysis, J. Nat. Dis., 7, 51-57, 1998

Sauchyn, D. J., Stroich, J., and Beriault A.: A paleoclimatic context for the drought of 1999-2001 in the northern Great Plains of North America, Geograph. J., 169, 158-167, 2003.

Sevinc, S. and Zekai, S.: Spatio-temporal drought analysis in the Trakya region, Turkey, Hydrol. Sci. J., 48, 809-820, 2003.

Wu, H. G. and Zhang, Z. M.: Meteorology, China Water Conservancy and Hydropower Publication House, 22-24, 1986.

Xu, G. M. and Zhou, H. L.: Principle of Earthquake, Science Publication House, 325-352, 1982.

Zhang, H. L.: Flood and Drought Disasters in China, China Water Conservancy and Hydropower Publication House, 282-283, 1997. 